\title{
Encefalitis a Citomegalovirus en pacientes con síndrome de inmunodeficiencia adquirida.
}

\author{
Encephalitis to Cytomegalovirus in patients with acquired immunodeficiency \\ syndrome. \\ Encefalite para Citomegalovírus em pacientes com síndrome de \\ imunodeficiência adquirida.
}

Facundo de Andrés Residente de Medicina Interna.

Diego Graña

Internista. Profesor Adjunto de Clínica

Médica.

Mario Torales

Internista. Infectólogo. Ex Profeso

Adjunto Clínica Médica. Servicio de Infectología - Hospital Pasteur.

Mabel Goñi

Internista. Inmunóloga. Profesor Director Clínica Médica.
Resumen: La encefalitis por citomegalovirus (CMV) es una enfermedad poco habitual en pacientes inmunocompetentes; no así en aquellos con compromiso de la inmunidad como los pacientes con SIDA, en especial con recuentos de linfocitos T CD4+ $<50 / \mathrm{mm} 3$. La encefalitis por CMV es una manifestación grave y potencialmente fatal, dado el amplio espectro clínico con el cual se manifiesta y el alto índice de sospecha requerido para su diagnóstico. Los estudios imagenológicos son orientadores pero no específicos, siendo los estudios de biología molecular por técnicas de PCR en el líquido cefaloraquídeo lo que permite establecer el diagnóstico de certeza. El tratamiento se basa en ganciclovir y foscarnet, asociados a la terapia antirretroviral. Presentamos tres casos clínicos con presentaciones heterogéneas, severo compromiso inmunitario; y donde la sospecha diagnóstica fue fundamental para establecer el tratamiento específico.

Palabras clave: Encefalitis, citomegalovirus, VIH-SIDA.

Abstract: Cytomegalovirus encephalitis (CMV) is an uncommon disease in inmunocompetent patients; not so in those with compromise of immunity as patients with AIDS, especially with counts of CD4 + T cells $<50 / \mathrm{mm} 3$. CMV encephalitis is a serious and potentially fatal manifestation, given the broad clinical spectrum with which it manifests and the high index of suspicion required for its diagnosis. The imaging studies are guiding but not specific, being the molecular study by PCR incerebrospinal fluid which allows to establish the diagnosis of certainty. The treatment is base dong an ciclovir and foscarnet associated an tiretroviral therapy. We present three clinical cases with heterogeneous presentations, severe immune compromise; and where the diagnostic suspicion was fundamental to establish the specific treatment

Key words: Encephalitis, Cytomegalovirus, HIV, AIDS.

Resumo: A encefalite por citomegalovírus (ECMV) é uma doença rara em pacientes imunocompetentes; não é assim naqueles com imunidade comprometida como pacientes com AIDS, especialmente com contagens de linfócitos T CD4 $+<50 / \mathrm{mm} 3$. O ECMV é uma manifestação séria e potencialmente fatal, dada a ampla gama do espectro clínico com o qual se manifesta e o alto índice de suspeita requerido para seu diagnóstico. Os estudos de imagem são orientadores, mas não específicos, sendo os estudos de biologia molecular por técnicas de PCR no líquido cefalorraquidiano, que permite estabelecer o diagnóstico de certeza. O tratamento é baseado em foscarnet de ganciclovids, associado à terapia anti-retroviral. Apresentamos três casos clínicos com apresentações heterogêneas, comprometimento imune severo; e onde a suspeita diagnóstica era fundamental para estabelecer o tratamento específico.

Palavras-chave: Encefalite, citomegalovírus, HIV-AIDS

Recibido: 11/02/2017 - Aceptado: 30/10/2017

Clínica Médica "2". Universidad de la República. Hospital Pasteur. ASSE. Montevideo, Uruguay. Correspondencia: E-mail: diegograna@vera.com.uy 


\section{Introducción}

El citomegalovirus (CMV) pertenece a la familia de los herpes virus humanos. ${ }^{(1)} \mathrm{La}$ encefalitis a CMV es una grave y potencial complicación del huésped inmunocomprometido, sobre todo con deterioro de la inmunidad celular y particularmente en los pacientes con síndrome de inmunodeficiencia adquirida (SIDA) con recuento de CD4 $<50 / \mathrm{mm}^{3}$. La infección por CMV es frecuente llegando hasta el $70 \%$ en algunas series; siendo la forma de infección por reactivación del virus..$^{(1,2,3)}$

El CMV es una infección oportunista frecuente en los pacientes con SIDA; estimándose que hasta un 30\% contraían la enfermedad en la era pre terapia anti retroviral (TARV), actualmente se estima en menos de 6 casos por 100.000/año.(2) La retinitis por CMV es la forma más frecuente de presentación, pudiendo afectar otros territorios como el tubo digestivo, pulmón y glándulas suprarrenales. A nivel del sistema nervioso central la manifestación más prevalente es la poliradiculopatía, la mielitis, ventriculitis, demencia y compromiso de pares craneanos; siendo el compromiso meningoencefálico poco habitual. ${ }^{(4)}$

EI CMV afecta a los pacientes con SIDA en etapas avanzadas, donde los linfocitos T CD4+ son menores a 50 células $/ \mathrm{mm}^{3}$. Otros factores de riesgo son: ausencia de TARV o que se encuentran en fallo terapéutico, carga viral elevada (> 100.000 copias $/ \mathrm{ml}$ ) e historia de infecciones oportunistas previas. El síndrome Inflamatorio de reconstitución inmune (SIRI) es otro factor de riesgo conocido en estos pacientes.

En cuanto al diagnóstico, la sospecha debe estar siempre presente en pacientes con severa inmunodepresión, que se presentan con síntomas neurológicos compatibles con infección a CMV y en donde los hallazgos imagenológicos son sugestivos pero no patognomónicos. ${ }^{(3,5-8)} \mathrm{El}$ estudio del liquido cefaloraquídeo (LCR) es la herramienta diagnóstica fundamental, confirma el diagnóstico a través de técnicas de biología molecular como la Reacción en cadena de la polimerasa (PCR) y descarta otras etiologías probables. ${ }^{(5-10)}$ El tratamiento se basa en la asociación de ganciclovir más foscarnet como inducción, existiendo otras drogas alternativas como cidofovir y valganciclovir. ${ }^{(1-3,9,10)}$

El objetivo de este trabajo es comunicar tres casos asistidos en un centro hospitalario de tercer nivel con diagnóstico de Encefalitis a CMV en etapa SIDA. Analizar las dificultades diagnósticas y de tratamiento en este grupo de pacientes así como remarcar la necesidad de un seguimiento y vigilancia más estrecho en esta subpoblación de pacientes VIH/SIDA con extrema vulnerabilidad.

\section{Caso clínico 1}

SM, 44 años. HIV-SIDA diagnosticado en contexto de Pneumocitosis pulmonar el mes previo a la consulta. No TARV. Linfocitos T CD4+ de $1 \mathrm{cél} / \mathrm{mm}^{3}$. Carga viral $700.000 \mathrm{copias} / \mathrm{ml}$. (datos del mes previo al cuadro actual).

Consultó por cuadro de 10 días de evolución dado por confusión mental, apatía y retracción conductual. Cefalea intensa holocraneana, agregando ataxia de tronco y lateropulsión a derecha. Sin fiebre. Al examen físico se presentaba desorientado, bradipsiquico, letárgico. Dermatitis seborreica. Paresia proximal de miembro superior derecho. Ataxia de tronco con lateropulsión derecha.

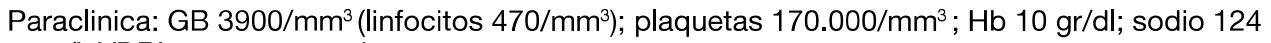
meq/l. VDRL sangre: negativo.

Resonancia nuclear magnética (RNM) de cráneo: aumento de la señal en T2 y Flair a nivel gangliobasal y esplenio del cuerpo calloso, sin restricción ni realce en la difusión sugestivo de proceso infeccioso. No se realizó espectometría (figura 1). 


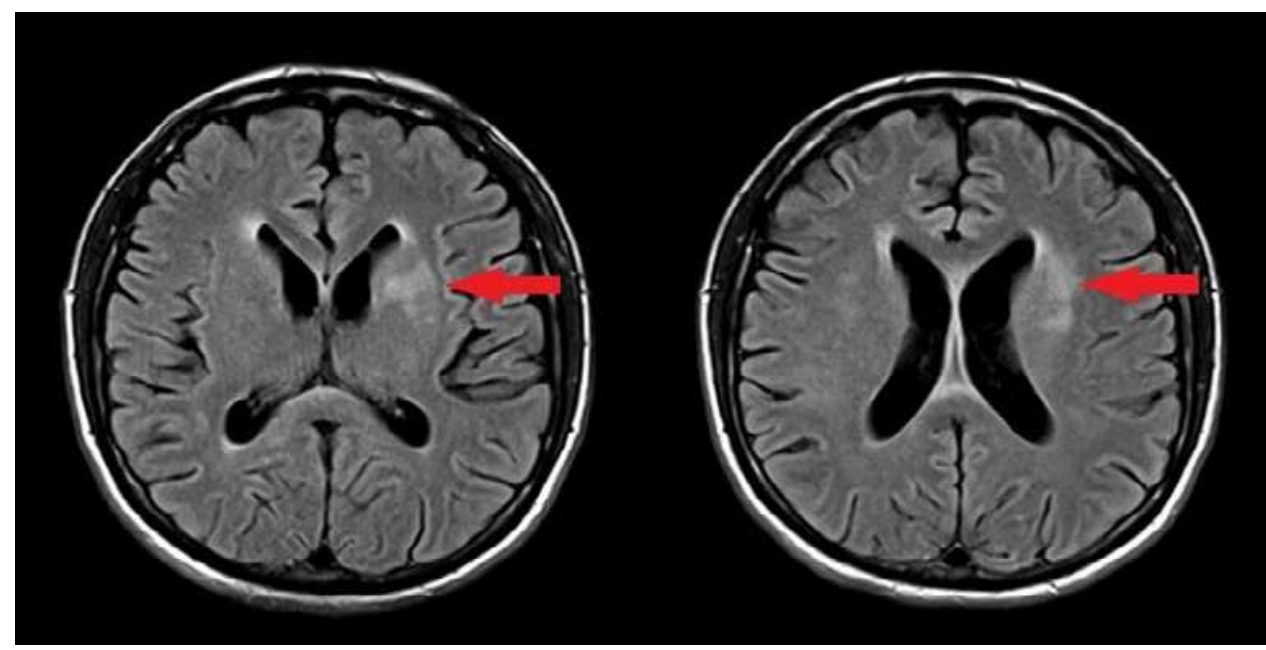

Figura 1: RNM de cráneo con zonas de

Estudio del LCR: líquido claro; proteínas $55 \mathrm{mg} / \mathrm{dl}$; glucosa39 mg/dl; sin glóbulos blancos. Tinta China: antígeno criptocococcico por ELISA negativo, cultivo para Cryptococcus neoformans negativo y VDRL negativo. Adenosíndeaminasa (ADA): 1. PCR para tuberculosis y grupo herpes virus (tipo 1, 2, 6 y EBV): negativos. Cultivo para hongos y bacteriológico negativos. PCR cualitativo para CMV positivo.

Frente a los hallazgos clínicos y de laboratorio, se planteó una encefalitis difusa producida por CMV.

Se realizó tratamiento con ganciclovir $5 \mathrm{mg} / \mathrm{Kg}$ endovenoso cada $12 \mathrm{hs}$ por 21 días como inducción, seguido de valganciclovir $900 \mathrm{mg}$ vía oral día como mantenimiento, con buena respuesta. Se inició TARV en base a zidovudina, tenofovir y efavirenz a las cuatro semanas del inicio del tratamiento para el CMV. Se realizó profilaxis para microrganismos oportunistas. Al alta presenta mejoría del nivel cognitivo, persistiendo las alteraciones en la marcha con lateropulsión a derecha.

\section{Caso clínico 2}

SM, 48 años. HIV- SIDA de 11 años de evolución. Presentó tuberculosis pulmonar, toxoplasmosis encefálica y criptococosis meníngea. Hidrocefalia secuelar crónica. TARV desde hace 10 años, plan actual con lamivudina, ritonavir y etravirine desde hace 3 años. Linfocitos T CD4+ de 7 $\mathrm{cél} / \mathrm{mm}^{3}$. Carga viral 300.000 copias $/ \mathrm{ml}$ (dato de 15 días previo al cuadro actual). Consultó por cuadro de 7 días de evolución dado por cefalea intensa holocraneana, tendencia al sueño y vómitos recurrentes. 24 hs previas al ingreso presentó dos episodios de convulsión tónicoclónicos generalizadas. Sin fiebre.

Al examen físico se presentaba vigil, bradipsiquico, con tendencia al sueño. Sin elementos de inmunodepresión clínica. El examen neurológico no reveló elementos de focalidad, no presentaba rigidez de nuca.

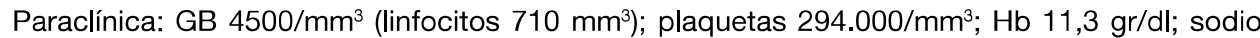
$139 \mathrm{meq} / \mathrm{l}$. VDRL en sangre negativo.

RNM de cráneo: Hiperintensidad en T2 y FLAIR que compromete ambos hemisferios cerebelosos, lóbulo temporal y frontal izquierdo, occipital derecho y ganglio basal bilateral. Realce nodular a nivel ganglio basal bilateral. Discreto realce ependimario. No se realizó espectometria (figura 2). 


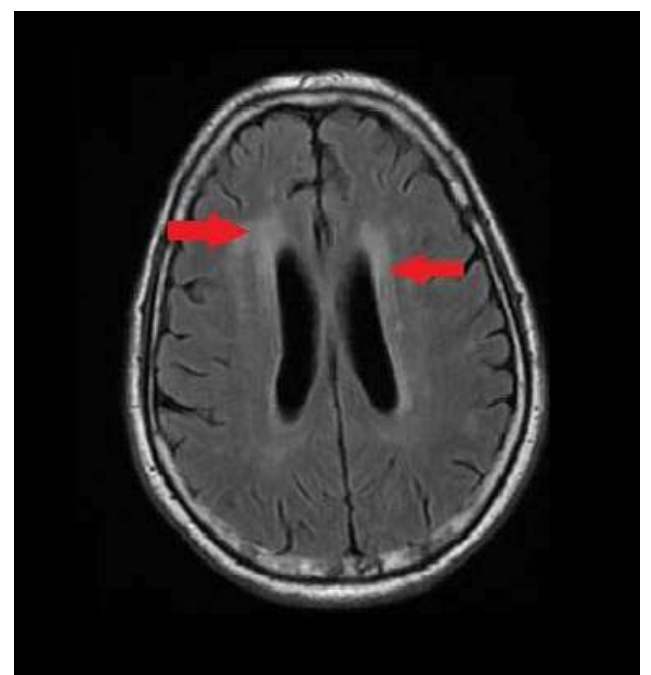

Estudio del LCR: líquido claro; proteínas $253 \mathrm{mg} / \mathrm{dl}$; glucosa 52mg/dl; GB: 15 monocitos. Tinta China y cultivo para Cryptococcus neoformans: negativo. VDRL negativos. ADA: 4. PCR para tuberculosis y grupo herpes virus (tipo 1, 2, 6 y EBV) negativos. Cultivo para hongos y bacteriológico: negativo. PCR cualitativo para CMV positivo.

Con los datos clínicos y paraclínicos encontrados se planteó una encefalitis Difusa producida por CMV.

Se realizó tratamiento con ganciclovir $5 \mathrm{mg} / \mathrm{Kg}$ endovenoso cada $12 \mathrm{hs}$ por 21 días como inducción, seguido de valganciclovir $900 \mathrm{mg}$ vía oral día como mantenimiento. Se asoció anticomiciales y se modificó el esquema de TARV previo, asociado a profilaxis para microrganismos oportunistas a las 4 semanas del cuadro actual. Presentó buena evolución clínica, con mejoría de los síntomas, sin reiterar convulsiones.

\section{Caso clínico 3}

SF, 44 años. Analfabeta. Consumo de sustancias psicoactivas. HIV/SIDA diagnosticado en contexto de Pneumocistosis pulmonar 6 meses previos. No cumple TARV. Linfocitos T CD4+ de $37 \mathrm{cé} l / \mathrm{mm}^{3}$. Carga viral 1.000 .000 copias $/ \mathrm{ml}$ (dato de 6 meses previo al cuadro actual). Consultó por cefalea holocraneana intensa de 7 días de evolución con heteroagresividad verbal y física. En apirexia. Al examen físico se presentaba lúcida. Lesiones compatibles con Herpes zoster multimetamérico en hemiabdomen derecho. Muguet oral. El examen neurológico no mostró rigidez de nuca ni elementos de focalidad neurológica.

Paraclínica: GB 5200/mm (linfocitos $700 \mathrm{~mm}^{3}$ ); plaquetas $189.000 / \mathrm{mm}^{3}$; Hb $12,1 \mathrm{gr} / \mathrm{dl}$; sodio $138 \mathrm{meq} / \mathrm{l}$. VDRL en sangre negativo.

Tomografía (TC) cráneo (sin contraste): Sistema ventricular aumentado de tamaño a predominio supratentorial (figura 3). No se pudo realizar RNM de cráneo. 


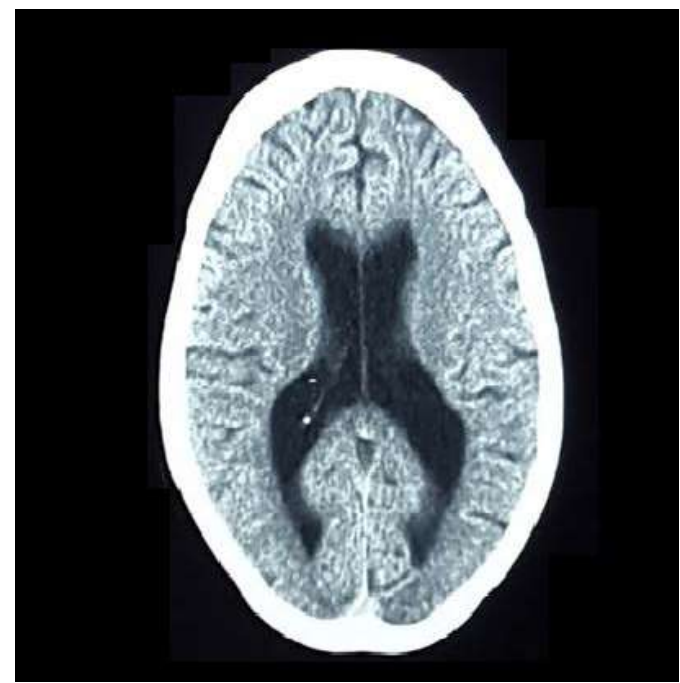

Estudio del LCR: Líquido claro; proteínas $95 \mathrm{mg} / \mathrm{dl}$; glucosa $43 \mathrm{mg} / \mathrm{dl}$; sin leucocitos. Tinta china, cultivo y antígeno para Cryptococcus neoformans: negativos. VDRL y bacteriológico: negativos. PCR para tuberculosis y grupo herpes virus (tipo 1,2, 6 y EBV): negativos. PCR para virus Varicela Zoster: negativo. PCR cualitativo para CMV positivo.

Con los datos encontrados se planteó encefalitis difusa producida por CMV.

Se realizó tratamiento con ganciclovir $5 \mathrm{mg} / \mathrm{Kg}$ endovenoso cada $12 \mathrm{hs}$ por 21 días. A las $72 \mathrm{hs}$

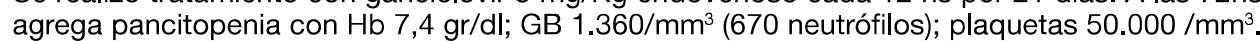
Se interpretó como toxicidad por fármacos, se disminuyó la dosis del ganciclovir a la mitad y se realizó reposición hematológica asociado a estimuladores medulares. Se continuó con valganciclovir como terapia de mantenimiento y se inició TARV en base a tenofovir, emtricitabine y efavirenz, además de profilaxis para microrganismos oportunistas tres semanas luego de iniciar tratamiento para CMV. Presentó mejoría clínica del cuadro neurológico y hematológico.

\section{Discusión}

La encefalitis por CMV ocurre en pacientes con severo compromiso de la inmunidad como lo reflejan el recuento de linfocitos CD4 de nuestros pacientes que fueron $1,7 \mathrm{y} 37 / \mathrm{mm}^{3}$ respectivamente, constituyendo este uno de los principales factores para su desarrollo.

El antecedente de infecciones oportunistas en este grupo de pacientes constituye otro factor de riesgo importante para la enfermedad por CMV, siendo la pneumocistosis pulmonar la más frecuente ${ }^{(2)}$ como se observa en dos pacientes de la serie (caso 1 y 3). Otra enfermedad que también se describe es la tuberculosis en sus diferentes formas presente en el restante caso (caso 2).

El compromiso de enfermedades oportunistas del SNC (criptococosis, toxoplasmosis) previas, se asocian a encefalitis por CMV en más de la mitad de los casos, como se observa en el caso 2. ${ }^{(1,11)}$

Es frecuente que la encefalitis a CMV ocurra en pacientes con infección previa o concomitante por CMV en otros parénquimas, principalmente retinitis. ${ }^{(1,2,8,12,13)} \mathrm{La}$ ausencia de manifestaciones clínicas puede deberse a lo inespecífico o asintomático ${ }^{(6,12)}$ del cuadro, por lo que se recomienda la valoración oftalmológica en forma sistematizada. Una de las complicaciones sistémicas más características del CMV lo constituye la adrenalitis, la cual se manifiesta habitualmente como hiponatremia de leve a moderada entidad (2), como sucedió en el caso clínico 1, si bien no podemos descartar otras causas para la misma.

Las manifestaciones clínicas de la encefalitis a CMV son variadas, la confusión y desorientación constituyen la forma de presentación más frecuente ( $90 \%$ de los pacientes) ${ }^{(2)}$, seguidas de la apatía, desinterés, bradipsiquia y cefalea holocraneana. La presencia de focalidad neurológica sólo se presenta en el $50 \%$ de los casos. ${ }^{(2)}$ La hiperreflexia difusa y la pérdida de memoria son hallazgos frecuentes de esta enfermedad. La demencia es una de las secuelas habituales de estos pacientes cuando el tratamiento no se realiza precozmente, e implica la necesidad de 
realizar al momento del diagnóstico y en la evolución test neuropsicológicos para su pesquisa. En la tabla 1 se presentan las características clínicas y tratamiento de los pacientes analizados.

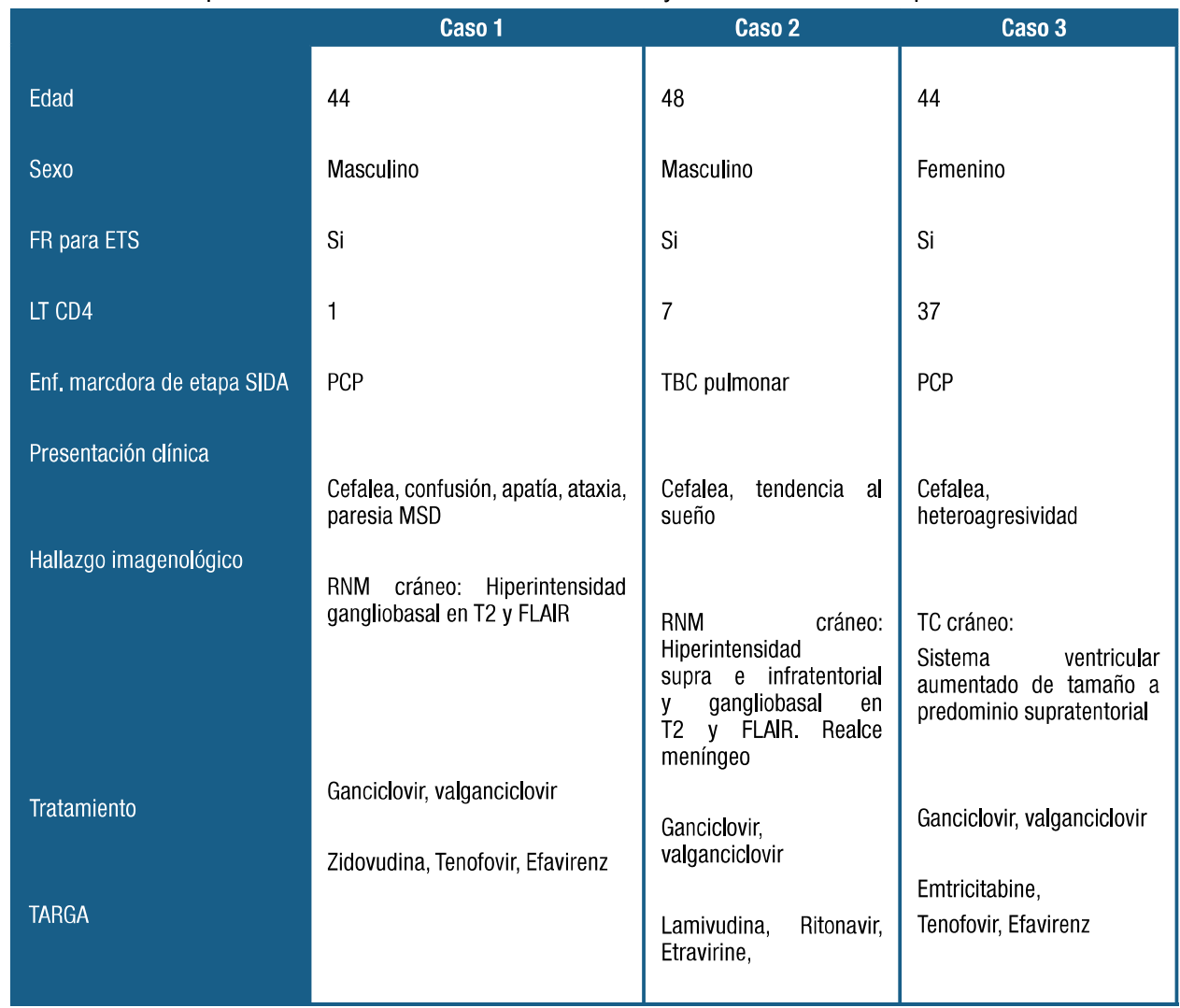

Aunque los hallazgos en la TC y RNM de cráneo poseen baja sensibilidad y especificidad para el diagnóstico de encefalitis a CMV, se han descripto imágenes que podrían sugerir la infección en un contexto clínico adecuado.

En la TC de cráneo puede apreciarse atrofia generalizada, ventriculomegalia e hipodensidad de la sustancia blanca periventricular que realza con el medio de contraste ${ }^{(1,2,9,14,15)}$, hallazgos presentes en el caso 3.

Las imágenes que pueden observarse en la RNM corresponden a ventriculitis con lesiones subependimarias hiperintensas en T2 y FLAIR con realce periférico lo que sugiere la 囚imagen en ojo de búho; alteraciones en la sustancia blanca con nódulos (hipo intensos en T1 e hiperintensos en T2), leucopatía difusa, atrofia focal y masa focal nodular. En este estudio adquiere importancia la técnica de difusión, la presencia de manchas brillantes con restricción en la difusión con tropismo ventricular son sugestivas de encefalitis por CMV. ${ }^{(16)}$ La RNM puede evidenciar aumento focal o difuso de la señal en T2 y FLAIR a predominio de la sustancia blanca periventricular. Es característico el realce ependimario y meníngeo tras la administración de gadolinio. ${ }^{(1,2,6,9,10,12-15}$ En la tabla 1 se resumen los principales hallazgos imagenológicos encontrados en nuestros tres casos, todos descritos en la literatura.

Es claro que, de los estudios de imagen la RNM constituye la técnica de elección.

El estudio del LCR no es específico para ECMV, presenta perfiles citoquímicos y celulares variables, generalmente es a liquido claro, raramente con pleocitosis y cuando ocurre es por linfocitos y monocitos, como pudo observarse en nuestros pacientes. La hipoglucorraquia es menos frecuente $(2,8,9,11-13,15,17-19)$. En nuestros pacientes no se constató la presencia de proteinorraquia ni glucorraquia y en el caso de celularidad aumentada fue a expensas de monocitosis. Cuando hay compromiso meningeo el perfil es el de una meningitis bacteriana, observándose hiperproteinorraquia en el $70 \%$ de los casos ${ }^{(11)}$.

El diagnóstico que permite la confirmación del CMV es a través de la detección del ADN viral en LCR por técnicas de PCR cualitativo o cuantitativo, que en un contexto clínico, inmunológico e imagenológico compatible permite realizar el planteo de encefalitis difusa por CMV. 
Si bien la sensibilidad y especificidad para el diagnóstico es mayor con la búsqueda de PCR cuantitativo o semi cuantitativo, la técnica empleada en nuestros casos posee sensibilidad y especificidad cercanas al $90 \% .(1,6,10-12,17)$

Existe escaso nivel de evidencia para las recomendaciones definitivas sobre el tratamiento de esta afección. La combinación de ganciclovir y foscarnet constituyen el esquema básico de inducción, a pesar de tener importantes efectos adversos. ${ }^{(20,21)}$

Hoy en día, ganciclovir es la primera línea de tratamiento, si bien existe el inconveniente creciente de las mutaciones UL97 y UL 54 que le restan eficacia. En estas situaciones el foscarnet es una alternativa en monoterapia, también se usa de igual forma en caso de leucopenia secundaria al uso de ganciclovir.

El cidofovir también se considera en el arsenal terapéutico.

Estos fármacos junto a la introducción de la TARV se han convertido en los pilares del tratamiento de la encefalitis por CMV.

En nuestra serie se utilizó ganciclovir durante tres semanas como terapia de inducción, de acuerdo a las recomendaciones de las guías nacionales e internacionales que sugieren un plazo de 14 a 21 días; seguido de valganciclovir como terapia de mantenimiento. No se utilizó foscarnet por no contar en nuestro medio con este fármaco.

En todos los casos se inició TARV según la guía nacional de tratamiento de pacientes VIH en plazos variables entre 3 y 4 semanas después de comenzado el tratamiento ${ }^{(20,21)}$.

Luego de 14 a 21 días de tratamiento con ganciclovir el paciente debe quedar con valgaciclivir asociado a TARV en forma precoz como tratamiento de mantenimiento.

Algunos autores sugieren discontinuar la terapia luego de que la enfermedad esté inactiva o en pacientes con reconstitución inmune sostenida (linfocitos T CD4+> $100 \mathrm{cel} / \mathrm{mm}^{3}$ ) por 3-6 meses bajo TARV. ${ }^{(12,15)}$

Como efecto adverso al tratamiento con ganciclovir, la paciente del caso 2 sufrió mielotoxicidad (pancitopenia) que fue tratada en base a reposición hematológica y factores de crecimiento medular. No requirió suspensión del tratamiento. Se trata de un evento frecuente, que afecta a más del $40 \%$ de los pacientes y que requiere ajuste de las dosis en forma habitual. ${ }^{(15)}$

La evolución natural de la encefalitis por CMV sin tratamiento es hacia el deterioro progresivo del estado mental y muerte, siendo el intervalo promedio desde el primer síntoma neurológico hasta el fallecimiento de 8,5 semanas. ${ }^{(2)}$ En una serie de 13 pacientes hospitalizados con diagnóstico de encefalitis por CMV con una media de 60 días de internación, se registró un $38 \%$ de mortalidad intrahospitalaria a pesar del tratamiento específico para CMV + TARV. La sobrevida fue sensiblemente menor en los pacientes con diagnóstico tardío. ${ }^{\left({ }^{(12)}\right.}$ Estos hechos marcan la necesidad de diagnóstico y tratamiento oportunos, lo que constituye un gran desafío en estos pacientes y refuerzan la necesidad de considerar el diagnóstico de encefalitis por CMV en todos aquellos pacientes con manifestaciones neurológica con severo compromiso inmunológico y hallazgos imagenológicos sugestivos ${ }^{(22) .}$

En nuestra serie de casos los diagnósticos se realizaron precozmente, no superando las $72 \mathrm{hs}$ entre el contacto con el paciente y el inicio del tratamiento específico, pudiendo esto explicar en parte la aceptable respuesta al tratamiento instituido.

\section{Conclusiones}

La encefalitis por CMV es una entidad infrecuente, que ocurre en pacientes con VIH-SIDA severamente inmunodeprimidos, siendo inhabitual en los inmunocompetentes. Dado el amplio espectro de manifestaciones clínicas se requiere un alto índice de sospecha clínica para su diagnóstico, lo que implica además la necesidad de una búsqueda sistematizada de este virus entre las agentes responsables de las manifestaciones neurológicas descritas. Se presentaron 3tres casos de esta afección con manifestaciones diferentes y en donde se estableció el diagnóstico en forma precoz, permitiendo establecer un tratamiento específico con la consiguiente buena respuesta clínica.

Los estudios de imagen son herramientas que sugieren la enfermedad pero son poco específicas, siendo la RNM la técnica de elección.

El pilar diagnóstico se basa en el análisis del LCR con la detección de ADN viral por técnicas de biología molecular como es la reacción en cadena de la polimerasa (PCR). 
El tratamiento en base a ganciclovir asociado a foscarnet en conjunto con TARV estableciendo la profilaxis secundaria con valgaciclovir.

La mortalidad sin tratamiento es muy alta, sin embargo bajo los esquemas terapéuticos actuales el pronóstico ha mejorado ostensiblemente como lo demuestra nuestra serie de casos.

\section{Bibliografía}

1- Arribas JR, Storch GA, Clifford DB, Tselis AC. Cytomegalovirus encephalitis. Ann Intern Med. 1996; 125: 577-587

2- Holland NR, Power C, Mathews VP, , Glass JD, Forman M, McArthur JC. Cytomegalovirus encephalitis in acquired immunodeficiency syndrome (AIDS) Neurology. 1994; 44: 507-514.

3- Morgello S, Cho ES, Nielsen S, Devinsky O, Petito CK. Cytomegalovirus encephalitis in patients with acquired immunodeficiency syndrome: an autopsy study of 30 cases and a review of the literature. HumPathol. 1997; 18: 289-297.

4- Bennett JE, Dolin R, Blaser MJ, ed. Mandell, Douglas y Bennett: Enfermedades infecciosas. Principios y práctica. 8va ed.Madrid: Elsevier, 2016.

5- Sanbonmatsu Gámez S, Pérez Ruiz M, Navarro Marí JM. Infección por citomegalovirus humano. Enferm Infecc Microbiol Clin. 2014; 32(Supl 1):15-22.

6- Steininger C, Puchhammer-Stöckl E, Popow-Kraupp T. Cytomegalovirus disease in the era of highly active antiretroviral therapy (HAART). J Clin Virol. 2006 Sep;37(1):1-9.

7- Prichard M1, Kern ER. The search for new therapies for human cytomegalovirus infections. Virus Res. 2011;157(2):212-21.

8- Pirskanen-Matell R, Grützmeier S, Nennesmo I, Sandström E, Ehrnst A. Impairment of short-term memory and Korsakoff syndrome are

common in AIDS patients with cytomegalovirus encephalitis. Eur J Neurol. 2009 Jan;16(1):48-53.

9- Anderson AM, Mosunjac MB, Corey AS, Fountain JA, Oshinski JN. Simultaneous typical and extraordinary imaging findings of AIDS-associated cytomegalovirus encephalitis. J Neurol Sci. 2011 Aug 15;307(12):174-7.

10- Kofteridis DP, Repa A, Anastasopoulos T, Barbounakis E, Gikas A, Karabekios S, et al. A case of human immunodeficiency virus infection disclosed by cytomegalovirus encephalitis. Int J Infect Dis. 2007 Jul;11(4):373-5.

11- Arribas JR, Clifford DB, Fichtenbaum CJ, Commins DL, Oowderly WG, Storch GA. Level of cytomegalovirus (CMV) DNA in cerebrospinal fluid of subjects with AIDS and CMV infection of the central nervous system. JInfect Dis 1995, 172:527-531.

12- Silva CA, Oliveira AC, Vilas-Boas L, Fink MC, Pannuti CS, Vidal JE. Neurologic cytomegalovirus complications in patients with AIDS: retrospective review of 13 cases and review of the literature. Rev Inst Med Trop Sao Paulo. 2010;52(6):305-310

13- Cohen BA. Prognosis and response to therapy of cytomegalovirus encephalitis and meningomyelitis in AIDS. Neurology 1996, 46:444-450.

14- Gottumukkala RV, Romero JM, Riascos RF, Rojas R, Glikstein RS.. Imaging of the brain in patients with human immunodeficiency virus infection. Top Magn Reson Imaging. 2014; 23(5):275-91.

15- Maschke M, Kastrup O, Diener HC. CNS manifestations of cytomegalovirus infections: diagnosis and treatment. CNS Drugs 2002; 16(5): 303-15.

16- Renard T, Daumas-Duport B, Auffray-Calvier E, Bourcier R, Desal HJ. Cytomegalovirus encephalitis: Undescribed diffusion-weighted imaging characteristics. Original aspects of cases extracted from a retrospective study, and from literature review. Neuroradiol 2016;43(6): 371-377.

17- Ginocchio CC. Laboratory diagnosis of human cytomegalovirus (HCMV) central nervous system disease in AIDS patients. Int J Antimicrob Agents. 2000 Dec;16(4):447-53.

18- Mhiri L, Kaabi B, Houimel M, Arrouji Z, Slim A. Comparison of pp65 antigenemia, quantitative PCR and DNA hybrid capture for detection of cytomegalovirus in transplant recipients and AIDS patients. $J$ Virol Methods 2007; 143:23-28.

19- Stanojevic M, Zerjav S, Jevtovic D, Salemovic D, Ranin J. CMV DNA in blood and CSF of HIV infected patients. Virus Res. 2002 Apr 23;85(1):117-22.

20- Uruguay. Ministerio de Salud Pública. Pautas de atención apacientes con VIH en Primer Nivel de Atención. Montevideo: MSP, 2016. 
21- Centers for Disease Control and Prevention, the National Institutesof Health, and the HIV Medicine Association of the Infectious Diseases Society of America. Guidelines for prevention and treatment of opportunistic infections in HIV infected adults an adolesents. AIDS info, 2014.

22- Almeida C, Silva A, Penalva de Oliveira L. Neurologic cytomegalovirus complications in patients with aids: retrospective Review of 13 cases and review of the literatura. Rev. Inst Med Trop. Sao Paulo 2010. 52(6):305-310. 